\title{
Diferentes Técnicas para el Alisado de Tensiones 3D
}

\author{
Mary J. Vergara, Sebastián Provenzano, Carlos Bloem y Rubén Chacón \\ Universidad de Los Andes, Facultad de Ingeniería, Escuela de Ingeniería Mecánica, \\ Grupo de Diseño y Modelado de Máquinas (DIMMA), La Hechicera, Mérida-Venezuela \\ (e-mail: vmary@ula.ve,prse@ula.ve,cbloem@ula.ve,rdchacon@ula.ve)
}

\begin{abstract}
Resumen
Se presenta la aplicación combinada de dos técnicas mejoradas para el alisado de tensiones por zonas: la Recuperación Superconvergente (Superconvergent Patch Recovery, SPR) y la Recuperación por Equilibrio (Recovery by Equilibrium Patches, REP). Estas técnicas mejoradas sobre hexaedros en problemas con dominio tridimensional, permiten obtener resultados más exactos al utilizar el método de los elementos finitos. Para analizar el comportamiento de la combinación de estas técnicas, se realiza un procedimiento h-adaptativo, utilizando el estimador de error Zienkiewicz-Zhu para determinar el error que involucra el campo de tensiones alisado. Los resultados de convergencia e índice de efectividad muestran que ambas técnicas por separado son análogas cuando las mallas tienen grados de libertad elevados.
\end{abstract}

Palabras clave: alisado de tensiones, estimador de error, superconvergent patch recovery, recovery by equilibrium patches

\section{Different Recovery Procedures for the Construction of Smoothed Stress in 3D}

\begin{abstract}
The combined application of two techniques improved for zone tension smoothing: Superconvergent Patch Recovery (SPR) and Recovery by Equilibrium Patches (REP), are analyzed. The techniques are improved on hexahedrons for three-dimensional domain problems which allow obtaining more exact results when using the finite elements method. To analyze the combination behavior of these techniques, an h-adaptive procedure is applied using the Zienkiewicz-Zhu estimator to determine the error that involves the smoothed stress field. The convergence results and efficiency index show that both techniques are analogous when the meshes have high degrees of freedom.
\end{abstract}

Keywords: stress smoothing, error estimator, superconvergent patch recovery, recovery by equilibrium patches 


\section{INTRODUCCIÓN}

Un progreso muy importante hacia las técnicas eficientes de postprocesamiento que mejoren la solución por elementos finitos (EF) ha sido presentada por Zienkiewicz y Zhu (1992) con el procedimiento de recuperación de tensiones "superconvergent patch recovery" (SPR), el cual esta basado en el alisado de tensiones por zonas, en éste una expansión polinómica describe las tensiones, usando un conjunto de elementos contiguos denominado "patch", alrededor de los nodos donde se desea realizar el alisado de tensiones. Esta expansión se hace usando mínimos cuadrados y las tensiones se calculan a partir de los valores de tensión evaluados mediante EF en los puntos de integración numérica. Después, varios autores presentaron mejoras como las realizadas por: Lee y Lo (1993), Blacker y Belystschko (1994), Wiberg y Abdulwahab (1993), Wiberg et al. (1994) y Ródenas (2002). Otra técnica que busca equilibrar las tensiones mejoradas en el patch de la misma forma que en el método de EF y con un procedimiento análogo al utilizado en el SPR, es el llamado "Recovery by Equilibrium Patches (REP)" propuesto por Booromand y Zienkiewcz (1997) y el cual muestra al igual que el SPR una gran robustez según Boroomand y Mossaiby (2005). El REP, también ha sido mejorado (REP-R) considerando las condiciones de contorno y se encuentra implementado individualmente igual que el SPR-R en Vergara (2002). Adicionalmente Lee et al. (1997), presentan otra técnica interesante "superconvergent stress recovery technique with equilibrium constraint" (LP), la cual es una combinación de las técnicas SPR y REP.

Este trabajo presenta la implementación combinada de las técnicas REP a SPR mejoradas y viceversa como una continuación del análisis presentado en Vergara et al. (2005), análogo a lo sugerido con la técnica LP, con la diferencia que éstas se implementan por separado y en dominios tridimensionales. Para definir las bondades de la composición, se utiliza el procedimiento h-adaptativo presentado en Vergara et al. (2007a); el cual es mas sencillo de aplicar con hexaedros que otros, como el presentado por Düster et al. (2007). Para aplicar el procedimiento h-adaptativo se utilizan técnicas para la estimación de error de discretización en subdivisión de hexaedros descritas en Vergara et al. (2007b). Finalmente se muestran los índices de efectividad y convergencia en un modelo con solución suave y otro con solución singular para obtener como resultado cual de las combinaciones de las técnicas estudiadas presenta mejores características para cada problema analizado.

\section{IMPLEMENTACIÓN DE LAS TÉCNICAS SPR-R y REP-R}

Las técnicas SPR-R y REP-R mejoradas son recientes, en ellas se cumplen exactamente las restricciones de tensión impuestas en los nodos del contorno con un pequeño coste computacional extra respecto a la técnica SPR y REP originales, y se encuentran implementadas con hexaedros en Vergara (2002). Estas se basan en su formulación original, como se puede detallar en Zienkiewicz y Zhu (1992) y Booromand y Zienkiewcz (1997), y su mejora puede resumirse según:

En el polinomio de interpolación de tensiones en el patch $\left(\boldsymbol{\sigma}_{p}^{*}\right)$ cada uno de sus componentes $\left(\sigma_{x}, \sigma_{y}, \sigma_{z}, \sigma_{x y}, \sigma_{x z}, \sigma_{y z}\right)$ se expresa según:

$$
\begin{aligned}
& \boldsymbol{\sigma}_{p i}^{*}=\alpha_{i 1}+\alpha_{i 2} x+\alpha_{i 3} y+\alpha_{i 4} z+\alpha_{i 5} x^{2}+\alpha_{i 6} y^{2}+\alpha_{i 7} z^{2}+\ldots \\
& i=x, y, z, x y, x z, y z
\end{aligned}
$$

donde: $\alpha_{i 1}$ son los coeficientes a determinar del polinomio por mínimos cuadrados.

El cumplimiento exacto de las restricciones de tensión impuestas en los nodos de ensamblado del patch puede ser forzado incorporando en el sistema de ecuaciones a resolver los valores conocidos de dichas restricciones. Para esto, en los patchs de nodos situados en el contorno los polinomios de interpolación de tensiones $\left(\boldsymbol{\sigma}_{p}^{*}\right)$ serán planteados en un sistema de referencia local de ejes $\xi, \eta$ y $\zeta$ con el origen en el nodo de ensamblado del patch y con los ejes orientados según las direcciones normal y tangenciales al contorno. Para plantear las ecuaciones de la técnica SPR en el nuevo sistema de coordenadas se realiza una traslación del sistema de coordenadas y dos giros, uno en $y$ y 
luego en $x$. Por lo tanto, las coordenadas locales $(\xi, \eta, \zeta)$ correspondientes a un punto de coordenadas globales $(x, y, z)$ se evaluarán, mediante la expresión:

$$
\left\{\begin{array}{l}
\xi \\
\eta \\
\zeta
\end{array}\right\}=\operatorname{Rot}\left\{\begin{array}{l}
x-x_{n} \\
y-y_{n} \\
z-z_{n}
\end{array}\right\}
$$

donde $\left(x_{n}, y_{n}, z_{n}\right)$ son las coordenadas globales del nodo de ensamblado del patch y Rot es la matriz de rotación, que viene dada por:

$\mathbf{R o t}=\left[\begin{array}{ccc}\cos \phi & 0 & -\operatorname{sen} \phi \\ -\operatorname{sen} \theta \operatorname{sen} \phi & \cos \theta & -\operatorname{sen} \theta \cos \phi \\ \cos \theta \operatorname{sen} \phi & \operatorname{sen} \theta & \cos \theta \cos \phi\end{array}\right]$

los ángulos $\phi, \theta$ definen la dirección normal al contorno respecto a los ejes globales $x, y, z$. Las tensiones en cualquier punto del patch expresadas en el nuevo sistema de coordenadas se evaluarán mediante la expresión:

$$
\left[\begin{array}{ccc}
\sigma_{\xi} & \sigma_{\xi \eta} & \sigma_{\xi \zeta} \\
\sigma_{\xi \eta} & \sigma_{\eta} & \sigma_{\eta \zeta} \\
\sigma_{\xi \zeta} & \sigma_{\eta \zeta} & \sigma_{\zeta}
\end{array}\right]=\operatorname{Rot}\left[\begin{array}{ccc}
\sigma_{x} & \sigma_{x y} & \sigma_{x z} \\
\sigma_{x y} & \sigma_{y} & \sigma_{y z} \\
\sigma_{x z} & \sigma_{y z} & \sigma_{z}
\end{array}\right] \operatorname{Rot}^{T}
$$

La transformación inversa para obtener $\sigma_{x}, \sigma_{y}, \sigma_{z}, \sigma_{x y}, \sigma_{x z}, \sigma_{y z}$ a partir de $\sigma_{\xi}, \sigma_{\eta}, \sigma_{\zeta}, \sigma_{\xi \eta}, \sigma_{\xi \zeta}, \sigma_{\eta \zeta}$ se calcula realizando una inversión planteada en la Ecuación 4. El nuevo sistema de referencia resulta conveniente para forzar el cumplimiento exacto de las restricciones de tensión impuestas en los nodos de ensamblado del patch, ya que las tensiones normales al contorno son conocidas. En el polinomio de interpolación de tensiones en el patch (ecuación 1) cada una de sus componentes en el nuevo sistema de coordenadas $\left(\sigma_{\xi}, \sigma_{\eta}, \sigma_{\zeta}, \sigma_{\xi \eta}, \sigma_{\xi \zeta}, \sigma_{\eta \zeta}\right)$ y sus correspondientes derivadas con respecto a dichas coordenadas puede ser expresada, por ejemplo para un polinomio de segundo orden, como:

$$
\begin{aligned}
& \boldsymbol{\sigma}_{p i}^{*}=\alpha_{i 1}^{*}+\alpha_{i 2}^{*} \xi+\alpha_{i 3}^{*} \eta+\alpha_{i 4}^{*} \zeta+\alpha_{i 5}^{*} \xi^{2}+\alpha_{i 6}^{*} \eta^{2}+\alpha_{i 7}^{*} \zeta^{2}+\alpha_{i 8}^{*} \xi \eta+\alpha_{i 9}^{*} \eta \zeta+\alpha_{i 10}^{*} \xi \zeta \\
& \frac{\partial \sigma_{p i}^{*}}{\partial \xi}=+\alpha_{i 2}^{*} \quad+2 \alpha_{i 5}^{*} \xi \quad+\alpha_{i 8}^{*} \eta \quad+\alpha_{i 10}^{*} \zeta \\
& \frac{\partial \sigma_{p i}^{*}}{\partial \eta}=\quad+\alpha_{i 3}^{*} \quad+2 \alpha_{i 6}^{*} \eta \quad+\alpha_{i 8}^{*} \xi+\alpha_{i 9}^{*} \zeta \\
& \frac{\partial \sigma_{p i}^{*}}{\partial \zeta}=\quad+\alpha_{i 4}^{*}+2 \alpha_{i 7}^{*} \zeta \quad+\alpha_{i 9}^{*} \eta+\alpha_{i 10}^{*} \xi \\
& i=\xi, \eta, \zeta, \xi \eta, \xi \zeta, \eta \zeta
\end{aligned}
$$

Ahora se puede forzar el cumplimiento exacto de las restricciones de tensión impuestas en los nodos de ensamblado considerando las expresiones (5) y (6) particularizadas en dicho nodo. Las expresiones anteriores evaluadas en el nodo de ensamblado del patch, de coordenadas $\xi=0, \eta=0, \zeta=0$ son: 


$$
\begin{aligned}
& \left.\sigma_{p i}^{*}\right|_{0}=\alpha_{i 1}^{*} \\
& \left.\frac{\partial \sigma_{p i}^{*}}{\partial \xi}\right|_{0}=\alpha_{i 2}^{*},\left.\quad \frac{\partial \sigma_{p i}^{*}}{\partial \eta}\right|_{0}=\alpha_{i 3}^{*},\left.\quad \frac{\partial \sigma_{p i}^{*}}{\partial \zeta}\right|_{0}=\alpha_{i 4}^{*}
\end{aligned}
$$

Si el valor de alguna de las tensiones o de sus derivadas es conocido en el nodo de ensamblado del patch, entonces se elimina el correspondiente valor del coeficiente $\alpha^{*}$ del sistema de ecuaciones planteado, condensando dicho sistema.

Las situaciones más habituales que se pueden plantear para forzar las condiciones de contorno son: Contorno con tracciones impuestas, si el nodo de ensamblado del patch se encuentra situado en un contorno donde se ha aplicado determinadas tensiones normales y tangenciales al contorno, entonces los valores de tensión normal y tangencial son conocidos en el nodo de ensamblado del patch. Contorno con restricciones de simetría, la condición de simetría en un contorno implica que la tensión tangencial ha de ser nula. En lo que se refiere a las tensiones en los casos estudiados, la condición de simetría implica simetría de dichas tensiones con respecto a la dirección normal al contorno, de tal forma que la derivada de las tensiones respecto a la dirección normal al contorno ha de ser nula y por lo tanto la condición de simetría en un contorno implica que las restricciones sobre dicho contorno son de ser de la forma dada en la ecuación (7). Lo anterior es válido para todos los nodos vértices que se encuentran ubicados en el contorno del dominio. Para incluir las ecuaciones de restricción en estos nodos, se sustituye directamente los valores de tensión conocidos sobre un sistema de referencia apropiado, que en este caso será nuevamente un sistema local ubicado en el nodo de ensamble del patch.

Una vez consideradas las mejoras de las técnicas SPR y REP originales, es necesario estudiar los parámetros que miden el comportamiento de la combinación de ambas en dos problemas con solución conocida, lo cual se presenta en el siguiente apartado.

\section{ÍNDICE DE EFECTIVIDAD Y CONVERGENCIA}

Para evaluar cada uno de los parámetros necesarios en la combinatoria de las técnicas SPR-R a REP-R y viceversa, se utilizan hexaedros lineales con integración completa para dos problemas estáticos, los cuales se describen a continuación:

a. Esfera de pared gruesa sometida a presión interna (ver Fig. 1) con: radio interno, $R_{1}=5 * 10^{-2}(\mathrm{~m})$, radio externo $R_{2}=20 * 10^{-2}(\mathrm{~m})$, Presión interna $P=1\left(\mathrm{~N} / \mathrm{m}^{2}\right)$, módulo de elasticidad $E=1000\left(\mathrm{~N} / \mathrm{m}^{2}\right)$, $v=0.3$ (adimensional), y energía de deformación exacta $\left\|\mathbf{u}_{\mathrm{ex}}\right\|^{2}=0.1308996939(\mathrm{Nm})^{1 / 2}$.

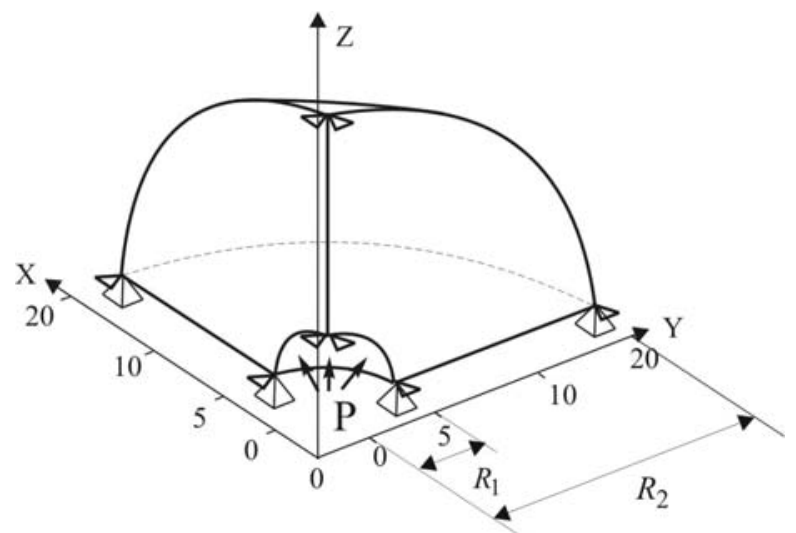

Fig. 1: Esfera de pared gruesa sometida a presión interna

b. Placa cargada a tensión con grieta lateral (ver Fig. 2) con: $\sigma=1000\left(\mathrm{~N} / \mathrm{m}^{2}\right)$, longitud de grieta de $a=0.6 * 10^{-2}$, en una placa de espesor $b=2 * 10^{-2}$, ancho $c=6 * 10^{-2}$, alto $d=1 * 10^{-2}, E=10^{7}\left(\mathrm{~N} / \mathrm{m}^{2}\right)$, 
$v=0.333,\left\|\mathbf{u}_{\mathrm{ex}}\right\|^{2}=0.112007(\mathrm{Nm})^{1 / 2}$. Para este problema sólo se analiza la combinación REP-R a SPR-R, debido a que al aplicar la técnica REP-R a ésta se obtiene un mejor resultado que con SPR$\mathrm{R}$, según Vergara et al. (2005).

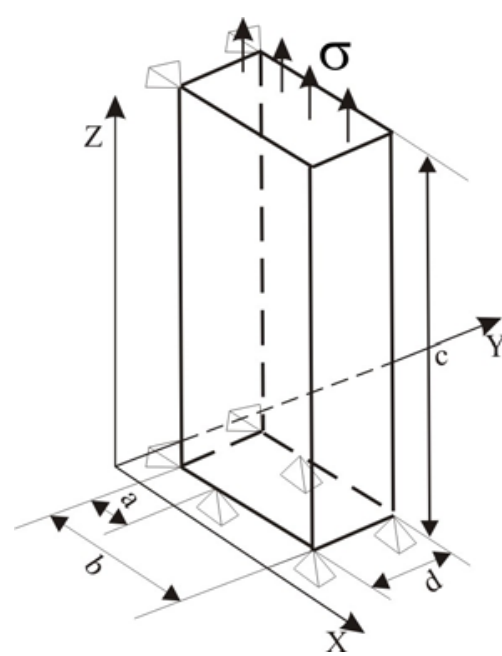

Fig. 2: Placa cargada a tensión con grieta lateral

Por otro lado, para estudiar el comportamiento de la combinatoria, se debe determinar el índice de efectividad y la velocidad de convergencia, según: impresión

$\theta=\frac{\left\|e_{e s}\right\|}{\left\|e_{e x}\right\|}$

donde $\left\|e_{e x}\right\|$ representa el error exacto y se determina según ecuación (9), $\left\|e_{e s}\right\|$ es el error estimado.

$\left\|e_{\mathrm{ex}}\right\|^{2} \approx \sqrt{\mathbf{u}_{\mathrm{ex}}\left\|^{2}-\mathbf{u}_{\mathrm{ef}}\right\|^{2}}$

$\left\|\mathbf{u}_{\mathrm{ex}}\right\|$ representa la norma energética de la solución exacta y $\left\|\mathbf{u}_{\mathrm{ef}}\right\|$ es la norma energética de la solución por elementos finitos que corresponde a la raíz cuadrada del doble de la energía de deformación, es decir:

$\mathbf{u}_{\mathrm{ef}} \|=\sqrt{\int \boldsymbol{\sigma}_{\mathrm{ef}}^{T} \mathbf{D}^{-1} \boldsymbol{\sigma}_{\text {ef }}} d V$

$$
e_{e s} \|=\sqrt{\left(\int_{V}\left(\boldsymbol{\sigma}^{*}-\boldsymbol{\sigma}_{e f}\right)^{T} \mathbf{D}^{-1}\left(\boldsymbol{\sigma}^{*}-\boldsymbol{\sigma}_{e f}\right) d V\right)}
$$

El valor de la energía de deformación exacta que es necesario para el cómputo del error ha sido obtenido en la grieta como el resultado de una malla muy refinada. Para estudiar la convergencia, se considera la formulación presentada en Vergara (2002), donde ésta se encuentra limitada por $p$ y $\lambda$ según el problema a valorar. Si la solución es suave, el valor de $\lambda$ es grande y entonces la velocidad de convergencia está limitada por el orden polinómico de los elementos, de tal manera que para problemas tridimensionales es válida la expresión 12.a, mientras que para el caso de la grieta el coeficiente $\lambda$ tiene un peso significativo en la convergencia y tiene un valor de 0.5 para una fisura casi cerrada. Considerando la singularidad en la solución se puede obtener una expresión para la velocidad de convergencia como se muestra en la ecuación (12.b). 

a. $v=-\frac{1}{3} p$
b. $v=-\frac{1}{3} \lambda$

Finalmente la velocidad de convergencia representa la pendiente de la curva $\eta \%=\log \eta \%$ contra $G D L=\log G D L$, para la cual es necesario definir error estimado relativo en la norma energética a nivel global como:

$$
n \%=\frac{e_{e s} \|}{{\sqrt{\mathbf{u}_{e f}{ }^{2}+\left.e_{e s}\right|^{2}}}^{2}} * 100
$$

\section{RESULTADOS Y DISCUSIÓN}

Al realizar un análisis de la evolución de la efectividad según la Fig. 3, se observa que al aplicar la técnica REP-R inmediatamente después de la SPR-R (REP-R+SPR-R) los resultados obtenidos hace que se encuentre el índice $\theta$ más cercano a la unidad hasta el tercer paso de malla, sin embargo para el último refinamiento el índice de efectividad es casi el mismo. La magnitud de la velocidad de convergencia es similar en los primeros refinamientos, sin embargo al aumentar los grados de libertad GDL aumenta hasta alcanzar un valor de 0.28 , el cual es muy cercano a la magnitud teórica esperada.
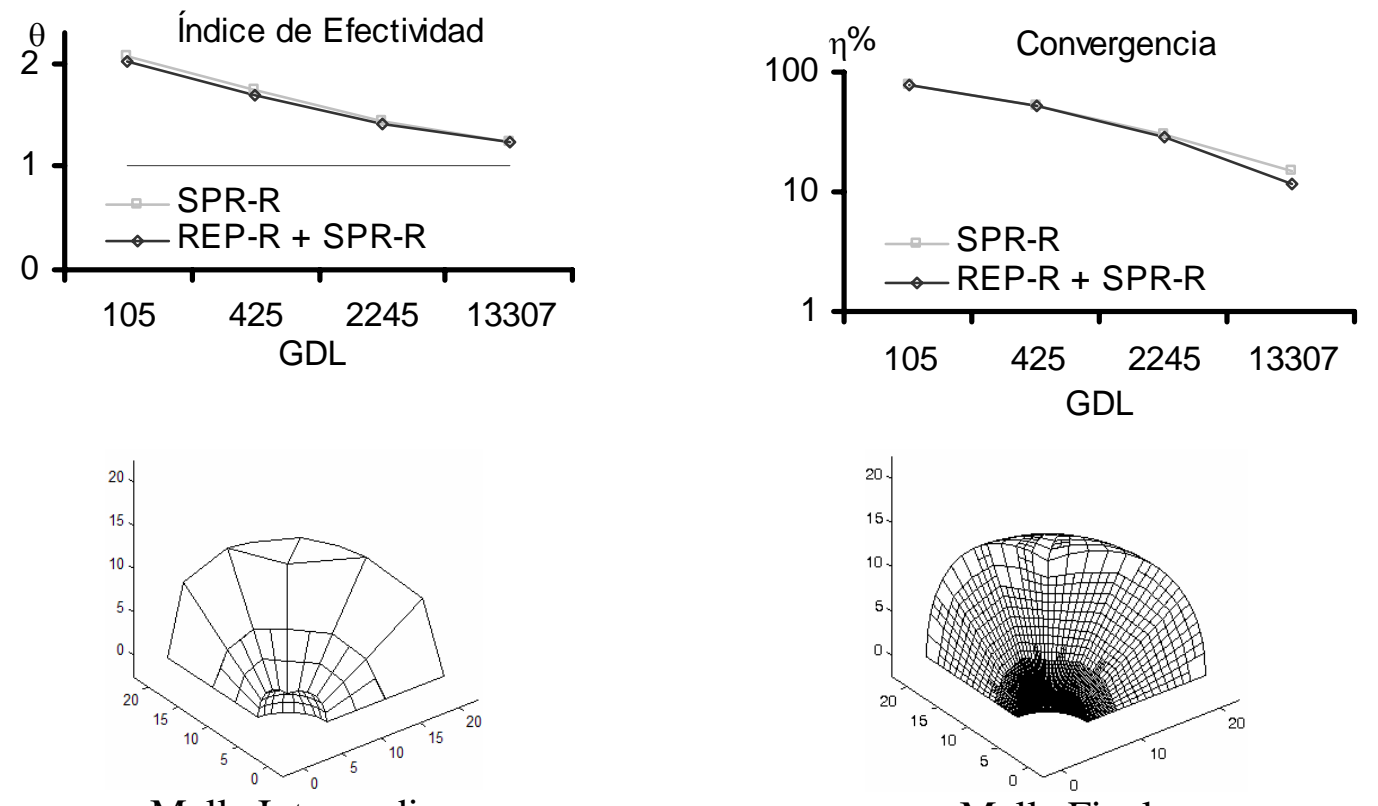

Malla Intermedia

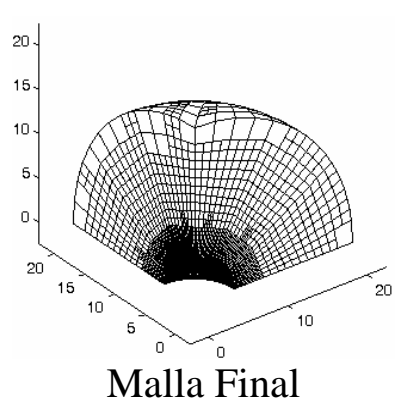

Fig. 3: Combinación en esfera SPR-R a REP-R

En la evolución del índice de efectividad en la Fig. 4, se observa que al aplicar la técnica SPR-R después de la REP-R ésta no mejora, ya que es superior con SPR-R+REP-R en todos los refinamientos de malla, no obstante para el último refinamiento el comportamiento es similar que el mostrado en la Fig. 1. La magnitud de la velocidad de convergencia es casi idéntica; en promedio es de 0.35 para todos los refinamientos.

En el problema de la grieta, el índice de efectividad tiende al valor deseado, de forma más cercana cuando se aplica SPR-R luego de REP-R, como se observa en la Fig. 5. Un comportamiento equivalente lo exhibe para la velocidad de convergencia, con un valor promedio de 0.30 para las tres últimas mallas. 

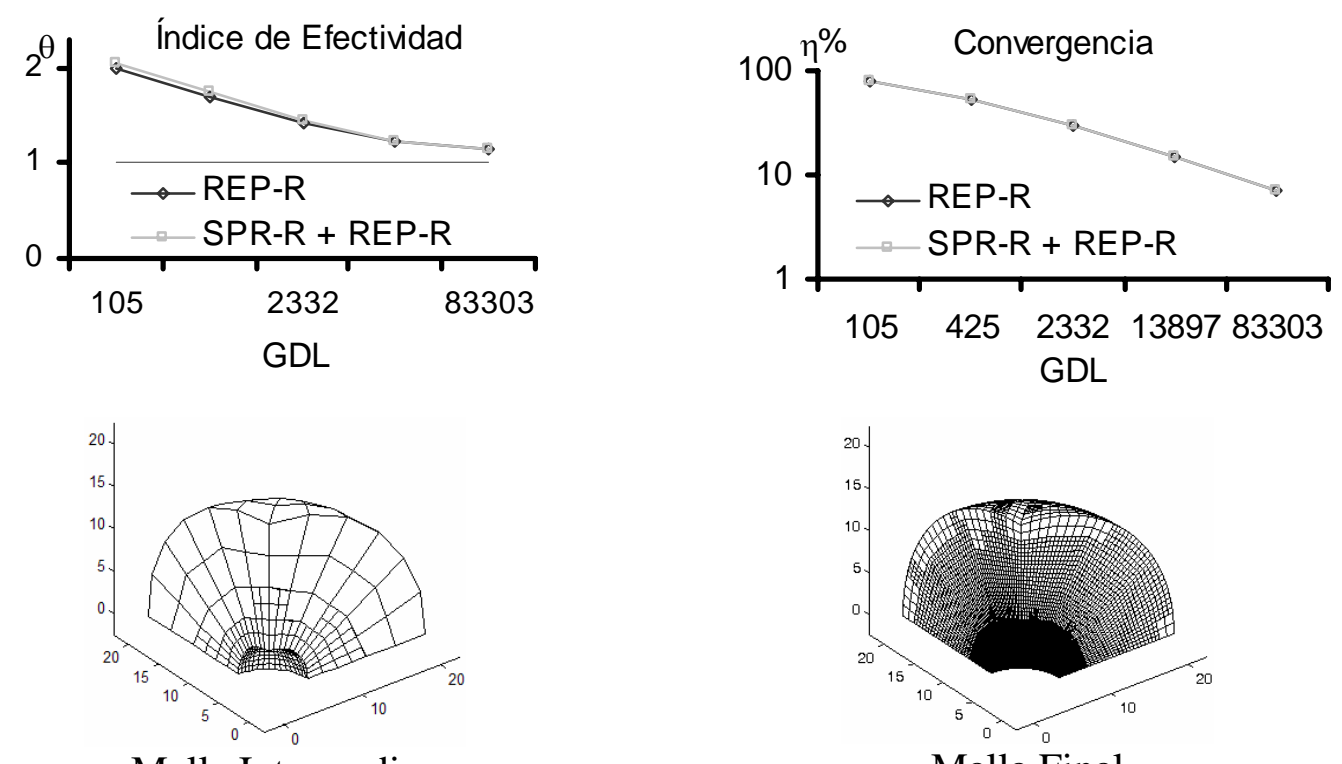

Malla Intermedia

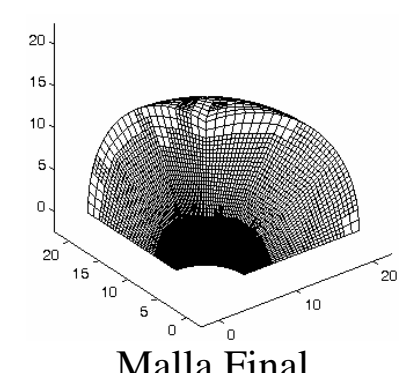

Fig. 4: Combinación en esfera REP-R a SPR-R
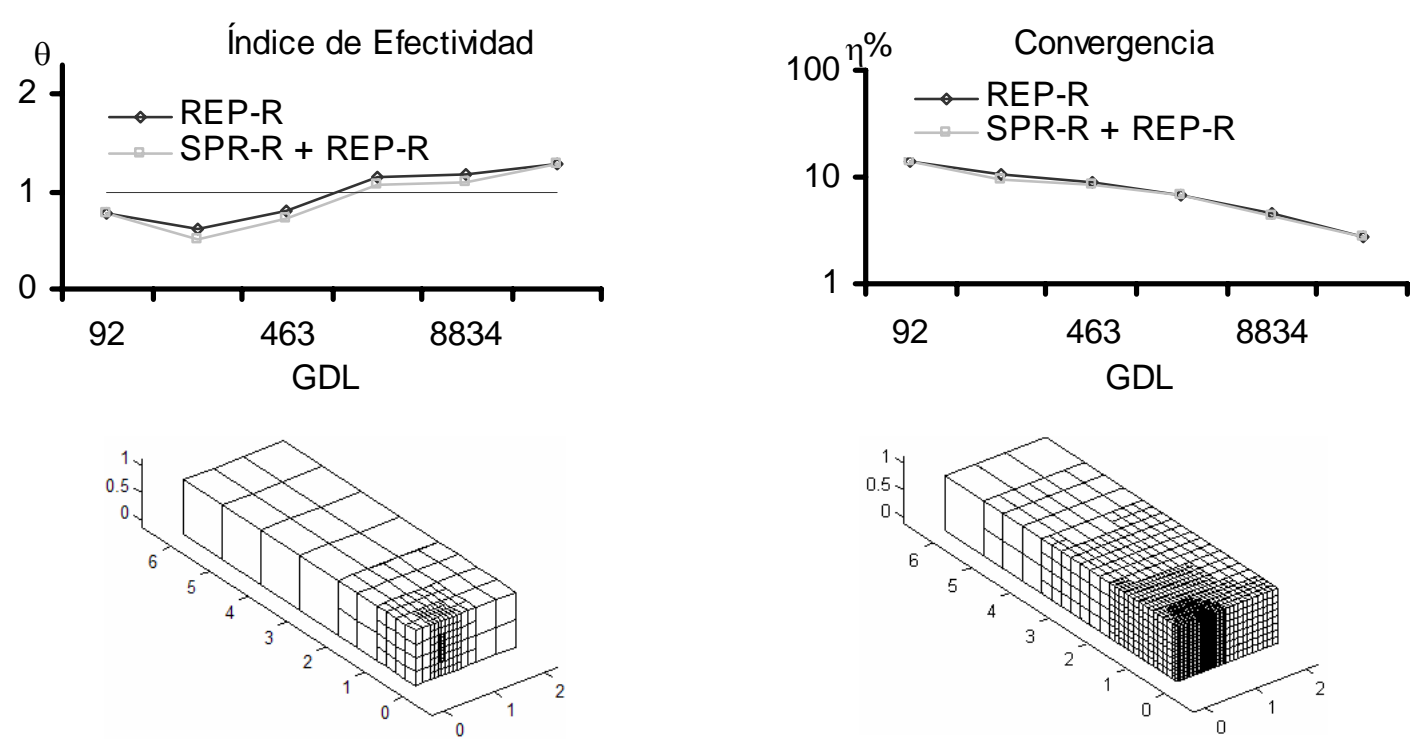

Malla Intermedia

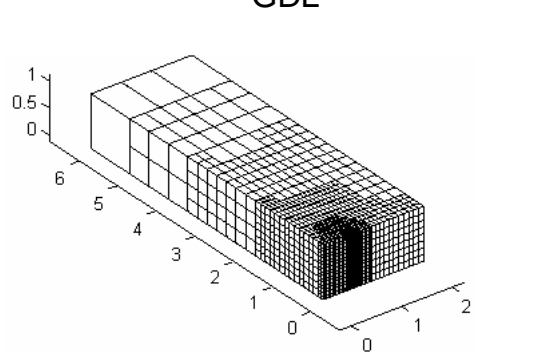

Malla Final

Fig. 5: Combinación en grieta REP-R a SPR-R

\section{CONCLUSIONES}

Los procedimientos combinados implementados para la reconstrucción del campo tensional presentan un comportamiento similar, ya que para cada caso, las magnitudes de los índices de efectividad tienden a la unidad cuando aumentan los GDL. Un comportamiento análogo lo presentan las velocidades de convergencia, las cuales tienden al valor teórico para cada caso estudiado. Para las técnicas combinadas implementadas en este trabajo, se concluye que para el caso de la esfera, la técnica combinada REP-R a SPR-R no mejora el índice de efectividad ni aumenta la velocidad de convergencia significativamente, mientras que al aplicar la técnica SPR-R a REP-R se mejora el índice de efectividad sin afectar significativamente la velocidad de convergencia. En el problema con solución singular, al aplicar la técnica SPR-R al procedimiento REP-R, se mejora el índice de efectividad, mientras que la velocidad de convergencia se mantiene casi constante. Es importante resaltar que según los resultados obtenidos, es indiferente utilizar una técnica de alisado mejorada (SPR-R o REP-R) o la combinación de ambas cuando se tienen varios refinamientos h-adaptativos. 


\section{AGRADECIMIENTOS}

Al CDCHT por el financiamiento otorgado a este trabajo designado bajo el código: I-801-04-02-A.

\section{REFERENCIAS}

Blacker, T. y T. Belystschko, "Superconvergente patch recovery with equilibrium and conjoint interpolant enhancements”, International Journal Numerical Methods in Engineering: 37 (1), 517-536 (1994).

Boroomand, B. y O. Zienkiewicz; "Recovery by Equilibrium in patches (REP)", International Journal Numerical Methods in Engineering: 40 (1), 137-164 (1997).

Boroomand B. y F. Mossaiby; "Generalization of robustness test procedure for error estimators. Part II: test results for error estimators using SPR and REP”, Int. J. Numer. Meth. Eng.: 64(1), 461-502 (2005)

Düster A., D. Scholz y E. Rank; "pq-Adaptive solid finite elements for three-dimensional plates and shells", Computer Methods in Applied Mechanics and Engineering: 197(4), 243-254 (2007).

Lee, C.K. y S. Lo; "Robust Implementation of convergent patch recovery technique". Computational Mechanics; 34(1), 1275-1280, (1993).

Lee, T., H. Park y S. Lee; "A Superconvergent Stress Recovery Technique with equilibrium Constrain", International Journal Numerical Methods in Engineering: 40(1), 1139-1160, (1997).

Ródenas, J. J.; "Error de Discretización en el cálculo de sensibilidades mediante el método de los elementos finitos", Ph.D. Thesis, Dpto. de Ingeniería Mecánica y Materiales, Universidad Politécnica de Valencia-Spain (2002).

Vergara, M.J.; "H-Adaptatividad en elementos finitos con refinamiento por subdivisión", Ph.D. Thesis, Dpto. de Ingeniería Mecánica y Materiales, Universidad Politécnica de Valencia-Spain (2002).

Vergara M. y otros cuatro autores; "Técnicas para la Estimación de Error de Discretización en Subdivisión de Hexaedros”, $8^{\circ}$ Congreso Iberoamericano de Ingeniería Mecánica, 20-25, Cusco, Perú, Octubre 23-25 (2007a).

Vergara M. y otros cuatro autores; "H-Adaptatividad en Elementos Finitos con Refinamiento por Subdivisión para Dominios 3D", 1er Congreso Internacional Científico/Técnico de Ingeniería CICTILUZ2007, 1013 1-17, Maracaibo, Venezuela, Noviembre 04-09 (2007b).

Vergara, M.J., S. Provenzano, L. Vergara y F. Rivas; "3D Problems Analysis using Recovery Stress", WSEAS Transactions on Computers: 4(4), 404-407, (2005).

Wiberg, N. y F. Abdulwahab; "Patch recovery based on superconvergent derivates and equilibrium", International Journal Numerical Methods in Engineering: 36(1), 2703-2724, (1993).

Wiberg, N., F. Abdulwahab y S. Ziukas; "Enhanced superconvergent patch recovery incorporating equilibrium and boundary conditions", International Journal Numerical Methods in Engineering: 37(1), 3417-3440, (1994).

Zienkiewicz O. y J.Z. Zhu; "The superconvergent patch recovery and a posteriori error estimates. Part 1. The recovery Technique”, International Journal Numerical Methods in Engineering: 33(1), 13311364, (1992). 